\title{
Investigating Linear Matrix Inequality Constraints in a 2.5MW DFIG Wind Turbine
}

\author{
Abbas Rasaienia ${ }^{1}$, Shima Hosseinian ${ }^{2}$ \\ 1 Department of Engineering, Islamic Azad University, \\ Garmsar Branch, Garmsar, Iran(Corresponding Author) \\ 1 prrasaienia@gmail.com \\ 2 Department of Electrical and computer, \\ Islamic Azad University, North Tehran Branch, Tehran, Iran \\ 2 shima_hosseinian70@yahoo.com
}

\begin{abstract}
The use of renewable energies has become widespread due to bioenvironmental concerns and a shortage of fossil fuels. In designing a robust controller for wind turbine system, variable speed is amongst one of the challenges to today's engineering. The wind energy transformation system includes complex aerodynamic and electrical components along with the unpredictable performance of wind speed and the other turbulence factors that render the existence of a robust controller necessary. In the present article, a robust controller has been proposed based on LMI method for variable-speed wind turbines with DFIG. To do so, the linear matrix inequality theories have been seminally explicated and the wind turbines and their nonlinear modeling have been subsequently introduced. Next, the linear model of the wind turbine system has been extracted based on the nonlinear model and offered within the format of M- $\Delta$ structure. Then, the linear matrix constraints have been defined for it and solved using MATLAB following which the feedback law is extracted and enforced on the system. The simulation results indicated that the controller has a good response.
\end{abstract}

Keywords: Wind turbine, Robust control, Linear matrix inequality

\section{INTRODUCTION}

During the past centuries, humankind has made use of wind as a source of energy. During the $17^{\text {th }}$ and $18^{\text {th }}$ centuries, the wind was considered as useful energy. During the late $19^{\text {th }}$ century, the first experiments were carried out for producing electricity by the use of wind but later on, little attention was paid to wind energy for generating power. It was with the expensiveness of the fossil fuels and oil that the attentions were once again directed towards renewable energies like the wind. Nowadays, wind energy is one of the important arms in generating power. However, in manufacturing and installing a wind turbine for generating power, energy generation, and such scales as output, cost, the effect on the power grid and so forth are of great importance.

Control system plays an important role in improving the output and performance of the wind energy conversion systems (WECS). The use of proper wind turbine controllers contributes to the maximization of the controlled power of the available wind energy and supports the machine and the structure in the course of extreme wind conditions. The complicacy of designing optimum controllers for wind energy conversion system is increased with the increase in the size of their power. To reach the maximum performance, the majority of wind turbines work in the variable speed (Vs) mode and variable pitch (Vp) angle.

In 2007, Bianchi used black-box method and model fitting technique based on system response data for identifying the wind energy conversion system's model [1]. There are many reasons for the use of DFIG wind turbines amongst which the possibility of the turbine energy's storage, reduction of stress in the mechanical structure, reduction in the acoustic noise, active and reactive power controllability and some others can be pointed out. In case of having no exact wind model and for system uncertainties, there is a need for robust controllers and use has been herein made of LMI method for the ease of calculations. In LMI method, the limitations of the constraints should be identified. One of the challenges in the available robust controllers is the improper response speed for the complex and time-consuming nature of problem solving. To overcome this problem, linear matrix inequality (LMI) has been suggested [4]. The controller of the robust phasic multivariate predictor model has been designed using LMI formulation via solving a convex optimization problem according to LMI conditions [5]. The output feedback $\mathrm{H}_{2} \mid \mathrm{H}_{\infty}$ controller designing method has been suggested based on LMI for power system stabilizers. The robust power system stabilizers outperform the conventional power system stabilizers and have the damping ability for various kinds of generators [6]. Quasi-LMI formula can be utilized for designing uncertainty and wind turbines with turbulence. This controller exhibits a relatively acceptable performance against chaos [7]. The continuous-time infinite horizon nonlinear square optimization control problem has been developed based on NSPS for a discontinuous-time state with the use of weighted 
matrix depending on states in cost function [8]. A robust control methodology has been proposed using minimum control from the set of LQG maxims for controlling the variable speed and variable pitch angles' classes of the wind turbines. These controllers display a good performance against chaos and reference speed variations [9]. The wind turbine's rotation speed and the output power are controlled by manipulating the pitch angle of the vanes (in a fixed generator torque). At first, a classic PID controller has been designed based on root place analysis whereas the second design belongs to a robust $\mathrm{H}_{2} \mid \mathrm{H}_{\infty}$ controller combined with $\mu$ based on DK replication algorithm. The results indicate that the robust controller guarantees the stability and performance of uncertainty systems [11].

The present study aims at investigating the inequality constraints of wind turbines and subsequent designing of robust controllers based on linear matrix inequalities. In the second part, the wind turbines and their generators are explicated. The third section deals with the principles of designing robust controllers. In the end, the method of designing robust controllers based on LMI is offered. The fourth section presents the results obtained from the designing method described in the third section. The conclusion and suggestions are also given in the fifth section.

\section{Introducing Wind Turbines:}

Wind turbines are classified into two groups of horizontal axis wind turbines (HAWT) and vertical axis wind turbines (VAWT) based on their rotation and rotation axis; they can be installed offshore and in the land. The main property of the HAWT is the high wind energy conversion output due to the vanes' designing style and access to stronger winds but they need a stronger tower for supporting their heavy weight because of which the costs of installation are increased. The VAWT have lower installation advantages such as lower wind energy conversion output due to the weakness of the wind in the lower section of the vanes and the resultantly more limited aerodynamic functioning of them. There is another form for classifying the wind turbines based on speed and power control methods. Wind energy conversion to fixed and variable speeds is also a classification method. Fixed-speed wind turbines (FSWT) rotate in a fixed velocity and the maximum energy conversion output can be attained only in given wind speed and the system output is decreased on the other wind speeds. Variable-speed wind turbine (VSWT) can provide the maximum energy conversion output in the entire span of a vast range of wind speeds. The turbine can continuously regulate the rotation speed in connection with wind speed. Another type of generator is a wind turbine system with an inductive generator (DFIG) [14]. For variable-speed systems with a speed change in a range from +30 to -30 percent of the synchronous speed, the doubly fed inductive generator can be a good solution. Amongst the advantages of DFIG, the followings can be pointed out: 1) having control over the reactive power; 2) having independent control over the active and reactive powers along with rotor flow control; 3) possibility of being magnetized from the rotor side; and, 4) the ability of generating reactive power that is delivered to stator. DFIG's modeling has been presented beneath:

\subsection{DFIG Model}

The fifth order dynamic equations of DFIG are obtained by transformation of three-phase inductive machine's equations to $\mathrm{d}-\mathrm{q}$ asynchronous rotation specifications [15]. The turbulence mode is defined as $w=$ $\left[v_{d s} v_{q s} i_{d r}^{r e f} i_{q r}^{r e f}\right]^{T}$ by selecting the state variables in the form of $x=\left[i_{d s} i_{q s} i_{d r} i_{q r}\right]^{T}$ wherein $i$ denotes current and $s$ denotes rotor and selecting the control variables in the form of $u=\left[v_{d r} v_{q r}\right]^{T}$ wherein $v$ denotes voltage (ref means reference). The observed outputs are $y=\left[i_{d r} i_{q r}\right]^{T}$ and the optimal outputs are $z=\left[i_{d r}^{r e f}-i_{d r} i_{d r}^{\text {ref }}-\right.$ $\left.i_{q r}\right]^{T} . \mathrm{L}_{\mathrm{s}}, \mathrm{L}_{\mathrm{r}}, \mathrm{L}_{\mathrm{m}}, \mathrm{W}_{0}, \Delta_{\mathrm{w}}, \mathrm{R}_{\mathrm{s}}$ and $\mathrm{R}_{\mathrm{r}}$ are the rotor's and stator's resistance, angular speed variations, initial angular speed, common, rotor and stator inductance, respectively. The following generalized form can be obtained:

$$
\begin{aligned}
& \dot{x}=A x+B_{1} w+B_{2} u \\
& z=C_{1} x+D_{12} w+D_{12} u \\
& y=C_{2} x+D_{21} w+D_{22} u
\end{aligned}
$$

It has the following matrices:

$$
\begin{aligned}
& A=\frac{1}{L_{m}^{2}-L_{r} L_{2}}\left[\begin{array}{cccc}
L_{r} R_{s} & -L_{r} L_{s} w_{0}+L_{m}^{2} \Delta w & -L_{m} R_{r} & L_{m} L_{r} \Delta w-\Delta L_{m} L_{r} w_{0} \\
-L_{m}^{2} \Delta w+L_{r} L_{s} w_{0} & L_{r} R_{s} & L_{m} L_{r} w_{0}-L_{m} L_{r} w_{0} & -L_{m} R_{r} \\
-L_{m} R_{s} & L_{m} L_{s} w_{0}-L_{m} L_{s} \Delta w & L_{s} R_{r} & L_{m}^{2} w_{0}+L_{r} L_{s} w_{0} \\
L_{m} L_{s} \Delta w-L_{m} L_{s} w_{0} & -L_{m} R_{s} & L_{r} L_{s} \Delta w-L_{m}^{2} w_{0} & L_{s} R_{r}
\end{array}\right] \\
& B_{2}=\left[\begin{array}{cr}
L_{m} & 0 \\
0 & L_{m} \\
-L_{s} & 0 \\
0 & -L_{s}
\end{array}\right], B_{1}=\left[\begin{array}{cccc}
-L_{r} & 0 & 0 & 0 \\
0 & -L_{r} & 0 & 0 \\
L_{m} & 0 & 0 & 0 \\
0 & L_{m} & 0 w_{0} 0
\end{array}\right]
\end{aligned}
$$


$C_{1}=\left[\begin{array}{cccr}0 & 0 & -1 & 0 \\ 0 & 0 & 0 & -1\end{array}\right], C_{2}=\left[\begin{array}{llll}0 & 0 & 1 & 0 \\ 0 & 0 & 0 & 1\end{array}\right]$

$D_{11}=\left[\begin{array}{llll}0 & 0 & 1 & 0 \\ 0 & 0 & 0 & 1\end{array}\right], D_{12}=D_{21}=D_{22}=0$

The active and reactive powers of the stator and rotor are:

$P_{s}=v_{d s} i_{d s}+v_{q s} i_{q s}$

$Q_{s}=v_{q s} i_{q s}-v_{d s} i_{d s}$

$P_{r}=v_{d r} i_{d r}+v_{q r} i_{q r}$

$Q_{r}=v_{q r} i_{d r}-v_{d r} i_{g r}$

$P=P_{r}+P_{s}$

$Q=Q_{r}+Q_{s}$

\subsection{Modeling Wind Turbines:}

At first, the turbine's torque is calculated:

$T=\frac{\rho A V^{3} c_{p}}{2 \omega_{r}}$

With $C_{p}=c_{1}\left(\frac{c_{2}}{\lambda_{l}} c_{3} \beta-c_{4}\right) e^{\frac{-c_{5}}{\lambda_{l}}}+C_{6} \lambda$ where $\mathrm{C}_{1}=0.5176, \mathrm{C}_{2}=116, \mathrm{C}_{3}=0.4 \mathrm{C}_{4}=5, \mathrm{C}_{5}=21, \mathrm{C}_{6}=0.0068$; A is the surface area of the turbine's brush and $\rho$ is the air density and $\mathrm{V}$ is the wind speed and is the rotor's speed.

The electrical torque takes the following form:

$T=L_{m} i_{q r} i_{d s}-L_{m} i_{d r} i_{q s}$

Now that the torque rates have been obtained, the rotor and generator's speeds can be written as shown below:

$J_{r} \dot{\omega}_{r}=T-k_{d t} \theta_{\Delta}-B_{d t} \dot{\theta}_{\Delta}-T_{e}$

$J_{g} \dot{\omega}_{g}=\frac{t_{d t}-k_{d t}}{N_{g}} \theta_{\Delta}-\frac{t_{d t} B_{d t}}{N_{g}} \dot{\theta}_{\Delta}-T_{g}$

$\dot{\theta}_{\Delta}=\omega_{r}-\frac{\omega_{g}}{N_{g}}$

\section{Designing the Controller:}

3.1. Designing State Feedback $H_{\infty}$ Controller:

In stability analysis problem, norm and $\mathrm{H}_{\infty}$ control synthesis problem, designing of robust state feedback controller and many of the other applications, use can be made of linear matrix inequalities. In vivid terms, such a vast area of use for linear matrix inequalities would be deemed illogical unless they are correctly and accurately solved. The main property of linear matrix inequality is that it defines a convex constraint according to variable decision-making vector. Due to the same reason, the feasible set is convex and can be obtained using convex optimization numerical algorithms, especially using a specific part of these optimization techniques called semidefinite programming as a generalization of linear programming. Nowadays, the majority of the applied algorithms depend on interior point methods [17-9].

Theorem $1\left(\mathrm{H}_{\infty}\right.$ norm): $\mathrm{H}_{\infty}$ norm of $\mathrm{G}(\mathrm{s})$ system is given as shown below [18\&28]:

$\left(\begin{array}{ccc}A^{T} P+P A & P B & C^{T} \\ B^{T} P & -\gamma I & D^{T} \\ C & D & -\gamma I\end{array}\right)<0$

$\mathrm{P}>0$

In this section, the linear system is dealt with as shown beneath:

$\left\{\begin{array}{l}\dot{x}=A x+B_{1} u+B_{2} \omega \\ z=C x+D_{1} u+D_{2} \omega\end{array}\right.$

For the linear system, the state feedback control law would be the following:

$\mathrm{U}=\mathrm{Kx}$

And, it gives the following closed-loop system:

$\left\{\begin{array}{l}\dot{x}=\left(A+B_{1} K\right) x+B_{2} \omega \\ z=\left(C+D_{1} K\right) x+D_{2} \omega\end{array}\right.$ 
For the linear system, the state feedback control law is designed so that:

$\left\|G_{z \omega}(s)\right\|_{\infty}<\gamma$

Where,

$G_{z \omega}(s)=\left(C+D_{1} K\right)\left(s I-\left(A+B_{1} K\right)\right)^{-1} B_{2}+D_{2}$

And, $\gamma$ is a positive scalar value [31].

Theorem 2: H-infinity problem has an answer if and only if there is a Matrix $\mathrm{W}$ and a definite symmetrical positive Matrix $\mathrm{X}$ in such a way that

$\left(\begin{array}{ccc}\left(A X+B_{1} W\right)^{T}+A X+B_{1} W & B_{2} & \left(C X+D_{1} W\right)^{T} \\ B_{B}^{T} & -\gamma I & D_{2}^{T} \\ C X+D_{1} W & D_{2} & -\gamma I\end{array}\right)<0$

When the matrices $\mathrm{W}$ and $\mathrm{X}$ are obtained, the state feedback control law is extracted from the following relation:

$\mathrm{K}=\mathrm{WX}^{-1}$

The above problem can be expressed in the following optimized form:

$\min \gamma$

S. t. $\mathrm{X}>0$

$\left(\begin{array}{ccc}\left(A X+B_{1} W\right)^{T}+A X+B_{1} W & B_{2} & \left(C X+D_{1} W\right)^{T} \\ B_{B}^{T} & -\gamma I & D_{2}^{T} \\ C X+D_{1} W & D_{2} & -\gamma I\end{array}\right)<0$

Moreover, if $\left(\mathrm{A}, \mathrm{B}_{1}\right)$ is found stabilizable, i.e.

$\left(A X+B_{1} W\right)^{T}+A X+B_{1} W<0$

The solution in relation (20) gives a stable asymptotic closed-loop system [31].

3.2. State Feedback Control by Pole Assignment:

The other method of designing state feedback control is through pole assignment. According to the structure given in relation [23], we know that there is a controller $\mathrm{k}$ for state feedback H-infinity control in such a way that

$\left\|G_{z \infty \omega}(s)\right\|_{\infty}<\gamma_{\infty}$ holds if and only if there is a matrix $\mathrm{W}_{\infty}$ and a Matrix $X_{\infty}>0$ in such a way that:

$\dot{x}=A x+B_{1} u+B_{2} \omega$

$z_{\infty}=C_{\infty} x+D_{\infty 1} u+D_{\infty 2} \omega$

$z_{2}=C_{2} x+D_{21} u$

$\left(\begin{array}{ccc}\left(A X_{\infty}+B_{1} W_{\infty}\right)^{T}+A X_{\infty}+B_{1} W_{\infty} & B_{2} & \left(C_{\infty} X_{\infty}+D_{\infty 1} W_{\infty}\right)^{T} \\ B_{B}^{T} & -\gamma_{\infty} I & D_{\infty 2}^{T} \\ C_{\infty} X_{\infty}+D_{\infty 1} W_{\infty} & D_{\infty 2} & -\gamma_{\infty} I\end{array}\right)<0$

When these conditions hold, the feedback gain matrix takes the following form:

$K=K_{\infty}=W_{\infty} X_{\infty}^{-1}$

But, considering the fact the pole assignment is going to be used for designing the controller, the pole assignment constraint would be holding if and only if there is a Matrix $W_{D}$ and a Matrix $X_{D}>0$ in such a manner that:

$Q \otimes X_{D}+S \otimes\left(A X_{D}+B_{1} W_{D}\right)+S^{T} \otimes\left(A X_{D}+B_{1} W_{D}\right)^{T}<0$

When these conditions hold, the feedback gain matrix takes the following form:

$K=K_{D}=W_{D} X_{D}^{-1}$

To reach an answer, there should be $\mathrm{XD}=X_{\infty}$ and $\mathrm{W}_{\mathrm{D}}=W_{\infty}$ in which case, the feedback gain would be in the following form:

$K=W_{\infty} X_{\infty}^{-1}=W_{D} X_{D}^{-1}$

Thus, to design the state feedback H-infinity controller through pole assignment, the relations (24) and (25) should be holding [31]. 


\subsection{Designing LMI-Based Robust Controllers:}

The objective of this section is designing a robust LMI-based controller for wind turbines. The wind turbine model along with DFIG generator and Drive Train System have been introduced and explained in the second section and their relations are mathematically shown beneath:

$$
\begin{aligned}
& \dot{x}=A x+B_{1 w} w+B_{1 u} u \\
& z=C_{11} x+D_{11} w+D_{12} u \\
& y=C_{21} x+D_{21} w+D_{22} u
\end{aligned}
$$

The uncertainty is considered as a parametric one that influences $C_{p}$ when being inserted in the wind speed relation. Robust control methods can preserve the robust system's performance and stability in the presence of uncertainty. Due to the same reason, they are largely taken into account in the industrial and uncertain systems. Therefore, system (29) is considered as an uncertain one wherein the matrices would have uncertain parameters.

$\left[\begin{array}{ccc}A_{\Delta} & B_{1 \Delta} & B_{2 \Delta} \\ C_{1 \Delta} & D_{11 \Delta} & D_{12 \Delta} \\ C_{2 \Delta} & D_{21 \Delta} & D_{22 \Delta}\end{array}\right]=\left[\begin{array}{ccc}A & B_{1} & B_{2} \\ C_{1} & D_{11} & D_{12} \\ C_{2} & D_{21} & D_{22}\end{array}\right]+\left[\begin{array}{c}B_{0} \\ D_{10} \\ D_{20}\end{array}\right] \Delta$

In the above relation, $\Delta$ points to uncertainty. The system in (29) can be considered equivalent to the following system.

$$
\begin{aligned}
& \dot{x}=A_{\Delta} x+B_{1 \Delta w} w+B_{1 \Delta u} u \\
& z=C_{11_{\Delta}} x+D_{11 \Delta} w+D_{12 \Delta} u \\
& y=C_{21 \Delta} x+D_{21 \Delta} w+D_{22 \Delta} u
\end{aligned}
$$

Here, efforts are made to downsize the uncertainty as much as possible. It is presumed in this method that the answer lies in the convex system and that the state variables are available because the designing method is based on state feedback. To design the robust state feedback controller in the face of the uncertainties, the following constraints should be taken into consideration [16].

$$
\left[\begin{array}{ccc}
A X+B_{1 u} W+(*) & * & * \\
B_{1 w}^{T}+D_{11}^{T} C_{11} X+D_{11}^{T} D_{12} W & -R & * \\
C_{11} X+D_{12} W & 0 & -I
\end{array}\right]
$$

It is worth mentioning that the sign $*$ in the above inequality relations point to the transpose of the matrix's element. Furthermore, in the above LMI, $=I=D_{11}^{T} D_{11}$. The convex answers of this LMI are equal to X and $\mathrm{W}$ provided that $\mathrm{X}=\mathrm{X}^{\mathrm{T}}>0$. After obtaining the $\mathrm{X}$ and $\mathrm{W}$ uncertainties through solving the above LMIs, the state feedback law can be determined in the form of relation [33]:

$\mathrm{u}=\mathrm{Fx}$

with the controller's gain being equal to $\mathrm{F}=\mathrm{WX}^{-1}$.

\section{Simulation:}

In this regard, it is necessary to offer a model of a wind turbine, DFIG and, Drive Train and eventually obtain the nonlinear model of the primary system via summing them up. Since the system has nonlinear factors, we are faced with a nonlinear system and, on the other hand, since the linear matrix inequality theory is specific to the linear systems, it is necessary to transform the nonlinear system to a linear system. To do so, use is made of Linmod instrument of Matlab. Next, before designing the controller, the open-loop system is investigated concerning its stability. Afterwards considering the explanations from the previous sections, the matrix constraints are defined for linear system and defined constraints are solved using Matlab. According to the decision-making variables obtained from solving the matrix constraints, feedback gain law is calculated. In the end, the attained state feedback law is inserted in the system's equations so that the closed-loop system can be obtained and finally the stability and step-response of it are investigated and compared with the results obtained from the open-loop state.

The wind turbine and its peripheral parameters used in the simulations can be found in Table 4-1, 4-2 and 4-3. These values have been excerpted from reference [16].

Table 4-1: Wind Turbine model's Parameters

\begin{tabular}{|l|c|l|c|}
\hline \multicolumn{1}{|c|}{ Parameter } & Value & \multicolumn{1}{c|}{ Parameter } & Value \\
\hline Blade radius & $\mathrm{R}=1$ & Broom surface area & $\mathrm{A}=10.387$ \\
\hline Wind turbine coefficient & $\mathrm{C}_{1}=0.5176$ & Wind turbine coefficient & $\mathrm{C}_{2}=116$ \\
\hline Wind turbine coefficient & $\mathrm{C}_{3}=0.4$ & Wind turbine coefficient & $\mathrm{C}_{4}=5$ \\
\hline Wind turbine coefficient & $\mathrm{C}_{5}=21$ & Wind turbine coefficient & $\mathrm{C}_{6}=0.0068$ \\
\hline Density of air & $\rho=1.225$ & & \\
\hline
\end{tabular}


Table 4-2: DFIG Generator Parameters

\begin{tabular}{|c|c|}
\hline Parameter & Value \\
\hline Stator Resistance & $\mathrm{R}_{\mathrm{s}}=0.023 \Omega$ \\
\hline Rotor Resistance & $\mathrm{R}_{\mathrm{r}}=0.016 \Omega$ \\
\hline Stator inductance & $\mathrm{L}_{\mathrm{s}}=0.18 \Omega$ \\
\hline Rotor Inductance & $\mathrm{L}_{\mathrm{r}}=0.16 \Omega$ \\
\hline Mutual inductance & $\mathrm{L}_{\mathrm{m}}=2.9 \Omega$ \\
\hline
\end{tabular}

Table 4-3: Driven Train model's Parameters

\begin{tabular}{|c|c|}
\hline Parameter & Value \\
\hline Low speed shaft inertia & $J_{r}=55 \times 10^{6}$ \\
\hline High speed shaft inertia & $J_{g}=390$ \\
\hline Girth rate & $N_{g}=95$ \\
\hline Hardness factor & $k_{d t}=2.7$ \\
\hline Attenuation coefficient & $B_{d t}=9.45$ \\
\hline efficiency drive train & $\eta=0.97$ \\
\hline
\end{tabular}

Here, the control inputs have been taken equal to $y=\left[\omega_{r} \omega_{g} T_{e}\right]^{T}, \omega=\left[V_{d s} V_{q s}\right]^{T}, u=\left[\beta T_{g} V_{d r} V_{q r}\right]^{T}$. It is observed with such selections that the system model is completely nonlinear. Thus, in order to make use of the linear matrix inequality theories, it is necessary to render system linear. To do so, Linmod instrument in Matlab is applied and the $\mathrm{u}$ inputs are modeled using inport and the $\mathrm{y}$ outputs are modeled by outport blocks. This way, the model is linearized and a seventh order system with seven state variables, three output variables and four input variables is obtained.

The state variables in this problem are equal to $=\left[\omega_{r} \omega_{g} i_{d s} i_{q s} i_{d r} i_{q r} T_{e}\right]^{T}$. The state equations are defined in the following form:

$$
\begin{aligned}
A & =\left[\begin{array}{ccccccc}
-1.8329 e-7 & 1.8086 e-9 & 0 & 0 & 0 & -5.2727 e-9 & -4.9091 e-8 \\
2.4741 e-4 & -2.6043 e-6 & 0 & 0 & 0 & 0 & 7.0688 e-5 \\
0 & 0 & 0.0152 & 84.8750 & -0.1915 & -3.0651 & 0 \\
3.4722 & -3.4603 & -84.7849 & 0.0152 & 306.5134 & -0.1916 & 0 \\
0 & 0 & -0.2754 & 344.8276 & 0.0119 & 5.6594 e 3 & 0 \\
-0.2155 & 0 & -344.8276 & -0.2754 & -5.6594 e 3 & 0.171 & 0 \\
1 & -0.0105 & 0 & 0 & 0 & 0 & 0
\end{array}\right] \\
B_{u} & =\left[\begin{array}{cccc}
-1,0190 e-7 & 0 & 0 & 0 \\
0 & 0.0026 & 0 & 0 \\
0 & 0 & 2.9 & 0 \\
0 & 0 & 0 & 2.9 \\
0 & 0 & -0.18 & 0 \\
0 & 0 & -0.18 & 0 \\
0 & 0 & 0 & 0
\end{array}\right], C_{21}=\left[\begin{array}{lllllll}
1 & 0 & 0 & 0 & 0 & 0 & 0 \\
0 & 1 & 0 & 0 & 0 & 0 & 0 \\
0 & 0 & 0 & 0 & 0 & 2.9 & 0
\end{array}\right], D_{22}=0
\end{aligned}
$$

The poles of the open-loop system are $1.7804 e^{-2} \pm 5.6403 e^{3} j, 1.1882 e^{-2} \pm 1.0381 e^{2} j,-1.3884 e^{-6} \pm$ $8.6090 e^{-4} j$ and $-1.0757 e^{-8}$ that is unstable for the fact that four poles fall on the right hand side of the Laplacian diagram.

\subsection{Designing LMI Controllers:}

To design the controller, two decision variables, namely $\mathrm{X}$ and $\mathrm{W}$, are taken into consideration that are $7 \times 7$ and $4 \times 7$ in dimensions. The two following LMIs are introduced in Matlab the toolbox of which is used for solving them.

$\mathrm{X}>0$

$\left[\begin{array}{ccc}A X+B_{1 u} W+(*) & * & * \\ B_{1 W}^{T}+D_{11}^{T} C_{11} X+D_{11}^{T} D_{12} W & -R & * \\ C_{11} X+D_{12} W & 0 & -I\end{array}\right]$ 
It is worth mentioning that the sign * in the above inequality relations points to the transpose of the matrix's element. Additionally, in the above LMI, $R=I-D_{11}^{T} D_{11}$. The convex answers of this LMI are equal to $\mathrm{X}$ and $\mathrm{W}$ on the condition that $\mathrm{X}=\mathrm{X}^{\mathrm{T}}>0$. The results of solving the above LMIs per each of these two decision variables have been given below:

$X=1 e+8^{*}=\left[\begin{array}{ccccccc}3.6532 & 0 & 0.1334 & -0.0311 & -0.0088 & 0.0020 & -0.9525 \\ 0 & 0 & 0 & 0 & 0 & 0 & 0 \\ 0.1334 & 0 & 3.6610 & -0.9522 & -0.2156 & 0.0588 & 0.5936 \\ -0.0311 & 0 & -0.9522 & 4.4033 & 0.0564 & -0.2731 & 0.4401 \\ -0.0088 & 0 & -0.2156 & 0.0564 & 0.0127 & -0.00035 & -0.0357 \\ 0.0020 & 0 & 0.0588 & -0.2731 & -0.0035 & 0.0169 & -0.0273 \\ -0.9525 & 0 & 0.5936 & 0.4401 & -0.0357 & -0.0273 & 3.3456\end{array}\right]$
$w=1 e 8^{*}\left[\begin{array}{ccccccc}0.0000 & 0.0000 & 0.0000 & -0.0000 & 0.0000 & -0.0000 & 0.0000 \\ 0.0009 & 3.0991 & 0.0931 & 0.0003 & 1.5582 & -0.1052 & 0.0058 \\ 0.0542 & -3.2542 & -0.0297 & 0.0217 & 0.3607 & 1.6292 & 0.2136 \\ 0.1038 & -0.3744 & -0.1080 & -0.3699 & 0.0238 & -2.1123 & 0.4042\end{array}\right]$

It is observed that the answers are completely feasible and Matlab's solver has made use of 57 replications. Moreover, the above two LMIs have been solved correctly because Matrix X is a positive and symmetrical definite matrix. This finding can be justified based on the eigenvalues obtained for it. The eigenvalues of the decision Matrix $\mathrm{X}$ have been given beneath:

Eigen value $(X)=\left\{4.4191 \times 10^{-5}, 4.4191 \times 10^{-5}, 8.6446 \times 10^{-6}, 2.0852,3470,4.5706,5.099\right\}$

After obtaining the $\mathrm{X}$ and $\mathrm{W}$ uncertainties via solving the above LMIs, the state feedback gain law can be obtained in the following form:

$\mathrm{F}=\mathrm{WX}^{-1}$

The values obtained for $\mathrm{F}$ are as shown below:

F

$=\left[\begin{array}{ccccccc}1.2302 e-05 & 1900.8836 & 0.00199 & -0.00013 & 0.0339 & -0.0020 & 1.2495 e-05 \\ 23.7644 & 157993890964.141 & 3092.1091 & -18981.7878 & 52760.2474 & -305940.9610 & 23.3018 \\ 2.0680 & -165902225337.117 & 2070.14071 & 39049.9156 & 34663.3678 & 629607.2937 & 0.9961 \\ 2.4365 & -19088770864.6607 & -773.1920 & -23466.3975 & -12636.9236 & -378245.2414 & 5.3938\end{array}\right]$

Now that the feedback gain law has been obtained, the controller's relation can be completed.

4.2. Closed-Loop Analysis of the Wind Turbine's DFIG:

As it was mentioned before, the controller's relation is in the state feedback form with its gain having been calculated in the previous section. To perform closed-loop analysis of the system, it is necessary to enter the state feedback controller into the system's cycle and observe and analyze its closed-loop answers. According to the fact that the controller is of the state feedback type:

$\dot{x}=A_{\Delta} x+B_{1 \Delta w} w+B_{1 \Delta u} F x$

$z=C_{11_{\Delta}} x+D_{11 \Delta} w+D_{12 \Delta} F x$

$y=C_{21 \Delta} x+D_{21 \Delta} w+D_{22 \Delta} F x$

Therefore, using x-factoring, the system's closed-loop matrix would be $A_{\Delta}-B_{1_{\Delta} u} F$. the eigenvalues of the closed-loop system are $-3.9825,07,0444,-158.90 \pm 51.381 \mathrm{j},-0.2180,-6.9579$ and -12.3685 .

Thus, it is observed that all seven eigenvalues related to closed-loop system have a negative integer part indicating that the closed-loop system is stable. In figures (4-6) to (4-8), the zero input answer pertains to the system's outputs. 

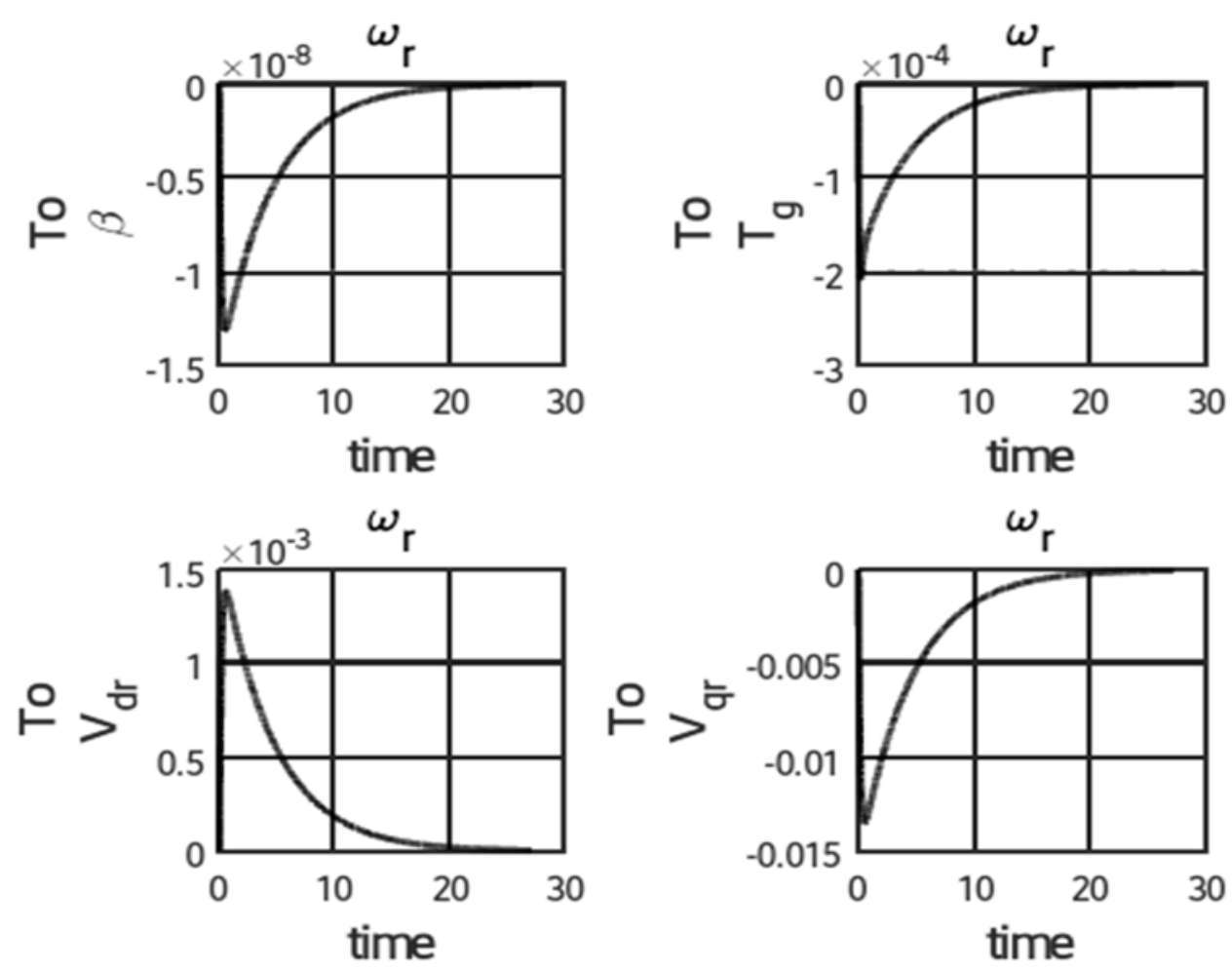

Figure (4-6): closed-loop system's step answer based on the second output of the inputs
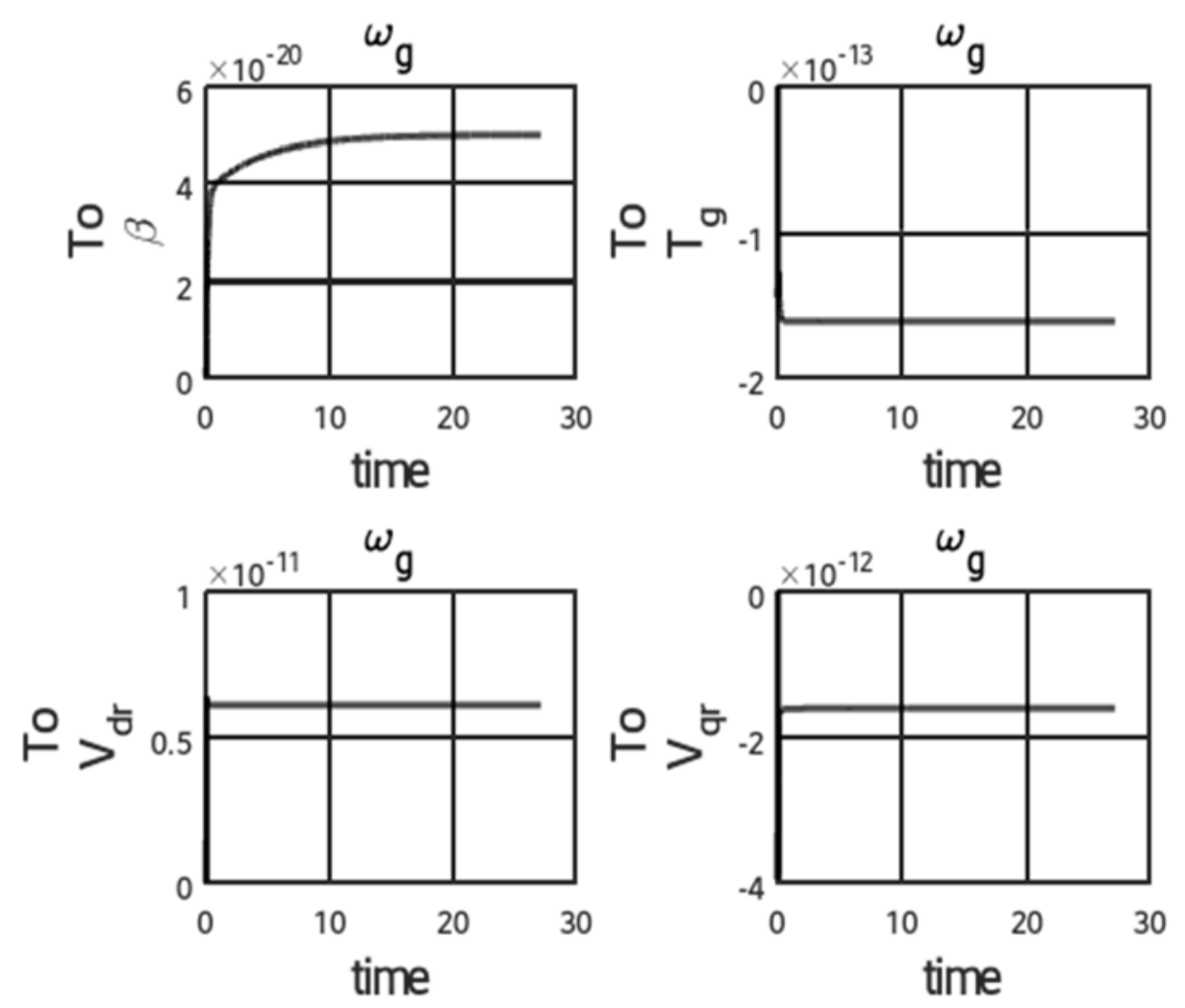

Figure (4-7): closed-loop system's step answer based on the second output of the inputs 

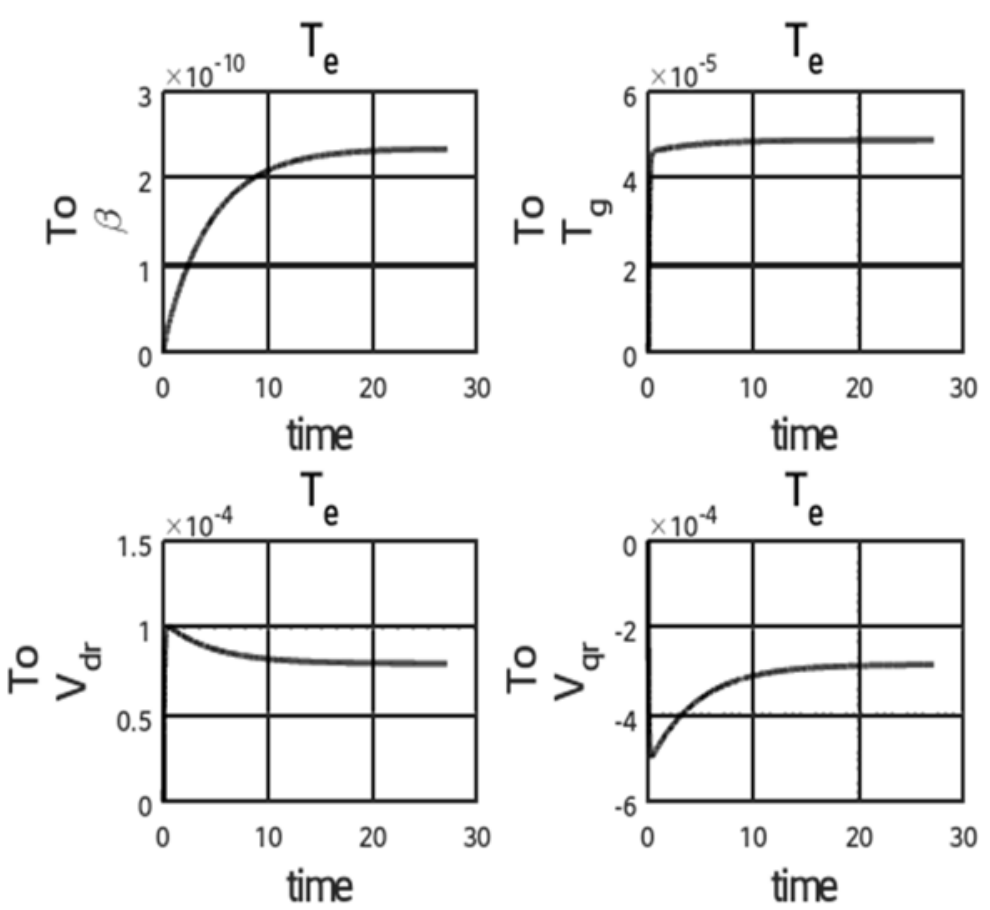

Figure (4-8): closed-loop system's step answer based on the third output of the inputs

It is observed that the controller has been able to stabilize all of the state variables of the system and regulate them on zero.

\section{Conclusion:}

In this article, linear matrix inequality control method was employed for controlling the stability of wind turbine systems with DFIG. The model that has been utilized for the wind turbine has four control inputs. Inn [15], the control inputs include $u=\left[V_{d r} V_{q r}\right]$ and signal designing has been used for robust controlling of them. However, in the current research paper, signals $\beta, T_{g}$ and have been inserted into the system's modeling process as the inputs respectively denoting the pitch angle and torque of DFIG. The constraints have been defined herein based on [16] that presents a wind turbine with parametrical uncertainty. However, in the present study's simulations, besides taking the turbine's mechanical torque into consideration, electrical torque has also been modeled and their outputs have been applied to extract the speeds' updating regulations. In [33], as well, the robust control theory has been applied for wind turbine but their model is simpler and neglects the generator's relations and equations and the uncertainty taken into account therein is only of parametrical type.

\section{References:}

[1] F. D. Bianchi, R. J. Mantz, and H. De Battista, The wind and wind turbines: Springer, 2007.

[2] A. Rasaienia and S. A. P. Razavi, "Design and Simulation of Robust Controller for Wind Turbine Using Linear Matrix Inequality."

[3] S. Bououden, M. Chadli, and H. R. Karimi, "Robust Predictive Control of a variable speed wind turbine using the LMI formalism," in 2014 European Control Conference (ECC), 2014, pp. 820-825.

[4] Y. Peng, Q. Zhu, and H. Nouri, "LMI based robust PSS for grid-connected DFIG wind turbines," in Modelling, Identification \& Control (ICMIC), 2013 Proceedings of International Conference on, 2013, pp. 147-152.

[5] K. Lahmadi, S. Aboulem, and I. Boumhidi, "LMI conditions to design a robust fuzzy controller for a wind generator," in Systems and Control (ICSC), 2016 5th International Conference on, 2016, pp. 313-318.

[6] Y. Zhang, Z. Liu, Z. Yang, and H. Si, "Robust control of wind turbines by using singular perturbation method and linear parameter varying model," Journal of Control Science and Engineering, vol. 2016, 2016.

[7] E. Kamal, A. Aitouche, and M. Bayart, "Fault-tolerant control of WECS subject to parametric uncertainties and sensor faults," in Control \& Automation (MED), 2011 19th Mediterranean Conference on, 2011, pp. 126-131.

[8] H. Moradi and G. Vossoughi, "Robust control of the variable speed wind turbines in the presence of uncertainties: A comparison between $H \circledR$ and PID controllers," Energy, vol. 90, pp. 1508-1521, 2015.

[9] B. E. Muhando and R. W. Wies, "Nonlinear \$ $\{\mid c a l ~ H\} \_\{\text {infty }\}$ \$ Constrained Feedback Control for Grid-Interactive WECS Under High Stochasticity," IEEE Transactions on Energy Conversion, vol. 26, pp. 1000-1009, 2011.

[10] S. Boyd, L. El Ghaoui, E. Feron, and V. Balakrishnan, Linear matrix inequalities in system and control theory vol. 15: Siam, 1994.

[11] I. Masubuchi, Y. Kamitane, A. Ohara, and N. Suda, "H® control for descriptor systems: A matrix inequalities approach," Automatica, vol. 33, pp. 669-673, 1997.

[12] P. Gahinet and P. Apkarian, "A linear matrix inequality approach to $\mathrm{H}{ }^{\circledR}$ control," International journal of robust and nonlinear control, vol. 4, pp. 421-448, 1994.

[13] R. Matusu, "Linear matrix inequalities and semidefinite programming: Applications in control," International Journal of Mathematical Models and Methods in Applied Sciences, 2014.

[14] G.-R. Duan and H.-H. Yu, LMIs in control systems: analysis, design and applications: CRC press, 2013. 